\title{
ANALYSIS OF COUPLING EFFECTS IN SEATED BODY BIODYNAMIC RESPONSES TO MULTI-AXIS VIBRATION
}

\author{
S. Mandapuram ${ }^{1}$, S. Rakheja ${ }^{1}$, P-E. Boileau ${ }^{2}$, \\ ${ }^{1}$ CONCAVE Research Center, Concordia University, Montréal, Canada \\ ${ }^{2}$ Institut de recherche Robert-Sauvé en santé et en sécurité du travail, Montréal, \\ Canada
}

\section{Introduction}

The biodynamic responses of seated body exposed to whole-body vibration (WBV) have been mostly studied under single-axis vibration, except for a few very recent studies. The responses to single-axis fore-aft and vertical vibration have shown considerable sagittal plane motions and magnitudes of cross-axis responses. The reported biodynamic responses to multi-axis vibration, however, suggest negligible coupled effects of multi-axis vibration ${ }^{1,2}$, although coupled motions of the body are clearly perceived by subjects and observed by experimenters ${ }^{3}$. This raises concerns over suitability of the data analysis method used in deriving multi-axis vibration biodynamic responses. This study examines the current data analysis method, primarily based on $\mathrm{H}_{1}$ estimator, and an alternate $\mathrm{H}_{3}$ estimator for analyses of responses to uncorrelated multiaxis vibration. The relative effectiveness of the $\mathrm{H}_{3}$ estimator in emphasizing the coupling effects of multi-axis vibration is demonstrated through analyses of apparent mass (APMS) and seat-to-head-transmissibility (STHT) data to dual-axis $(x z)$ vibration.

\section{Methods}

Majority of the studies have reported biodynamic responses derived using the $\mathrm{H}_{1}$ method based on cross-spectral density (CSD) of the measured signals, such that:

$$
H_{k l}=\frac{s_{a_{k} b_{l}}}{s_{a_{k}}} ; k=x, y, z \text { and } l=x, y, z
$$

Where $H_{k l}(j \omega)$ defines the complex direct $(k=l)$ or cross-axis $(k \neq l)$ APMS or STHT function, $S_{a_{k} b_{l}}$ is CSD of the response (force measured at the driving-point or the head acceleration along direction $l, l=x, y, z)$ and the input acceleration $a_{k}(k=x, y, z)$ with autospectral density of $S_{a_{k}}$. A few studies have also employed power-spectral density (PSD) or root-mean-square (RMS) methods, which yield identical magnitude results under single axis vibration. Using the linear system theory, the total response along each axis under multi-axis vibration can be considered as the sum of both the direct- and cross-axis responses to individual axis, such that:

$$
\bar{H}_{k}=\frac{s_{a_{k} b_{k}}}{s_{a_{k}}}=\sum_{l} \frac{s_{a_{k} b_{k I}}}{s_{a_{k}}}
$$

Where $\bar{H}_{k}$ is total biodynamic response along axis $k, S_{a_{k^{2}} \bar{b}_{k}}$ defines CSD of total response to input along $k, S_{a_{k} b_{k l}}$ is CSD of either direct $(k=l)$ or cross $(k \neq l)$ component of response along $k$ to single axis excitation along $l$ and $b_{k l}$ is response along $k$ due to excitation along $l$. In multi-axis experiments, the vibrations applied along individual axis are uncorrelated $\left(S_{a_{k} a_{I}}=0\right)$, which would lead to $S_{a_{k} b_{k l}}=0 \quad(k \neq l)$. Consequently, the biodynamic responses derived using $\mathrm{H}_{1}$ method would not account for the contributions of the cross- 
axis components observed under single axis vibration. The PSD method considers autospectral density of response alone and could thus appropriately account for cross-axis components, while it would not provide the phase data. Alternatively, the $\mathrm{H}_{3}$ estimator combines the advantages of both the $\mathrm{H}_{1}$ and PSD methods, by incorporating the crossaxis components and providing the phase information, and is given by:

$$
H_{k}=\sqrt{\frac{s_{a_{k} \delta_{k}}}{s_{a_{k}}} \frac{s_{\delta_{k}}}{s_{\delta_{k} a_{k}}}}
$$

The suitability of $\mathrm{H}_{3}$ estimator is investigated through analyses of STHT and APMS data acquired with 9 seated subjects exposed to individual $x$ and $z$-axis and combined $x z$ axes.

\section{Results}

Fig. 1 compares the mean vertical APMS and STHT responses obtained under single-axis vibration using the $\mathrm{H}_{1}$ method, and under dual-axis vibration using $\mathrm{H}_{1}$ and $\mathrm{H}_{3}$ estimators. The dual-axis responses derived using $\mathrm{H}_{1}$ are quite comparable to the singleaxis responses, particularly in APMS, as reported ${ }^{1,2}$. The $\mathrm{H}_{3}$ method, on the other hand, shows greater coupling effect of the dual-axis vibration by emphasizing contributions due to cross-axis responses observed under single-axis vibrations, which are evident at lower frequencies. The results obtained using $\mathrm{H}_{3}$ method also support the response attained through superposition of direct and cross axis responses to single-axis vibration ${ }^{3}$. The $\mathrm{H}_{3}$ method is thus considered better suited for the analysis of biodynamic response data to uncorrelated multi-axis vibration and the study of coupling effects.
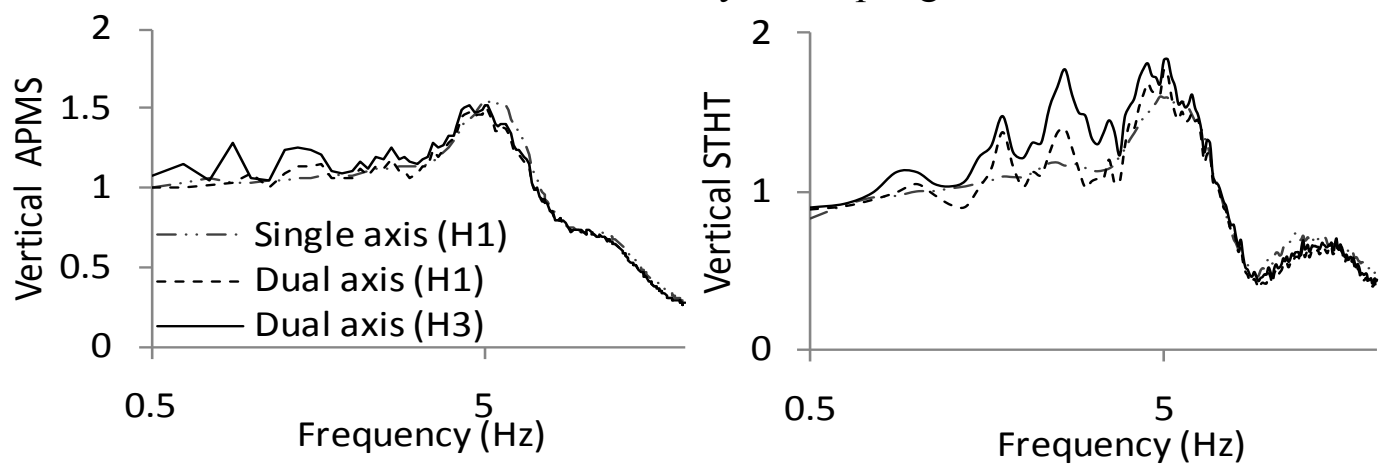

Fig. 1: Comparisons of single- and dual-axis vertical APMS and STHT responses.

\section{References}

1. Hinz B, Blüthner R, Menzel G, Rützel S, Seidel H and Wölfel Horst P (2006). Apparent mass of seated men - Determination with single and- and multi-axis excitation at different magnitudes. J. Sou \& Vib, 298, 788-809.

2. Mansfield N J and Maeda S (2007) The apparent mass of the seated human exposed to single-axis and multi-axis whole-body vibration. J. Biomechanics, 40, 2543-2551.

3. Mandapuram S, Rakheja S, Boileau P-É, Maeda S and Shibata N (2009) Apparent mass and seat-to-head transmissibility responses of seated occupants under single and dual axis horizontal vibration. Prod. $4^{\text {th }}$ Whole-Body Vib Injury Conf, 33-34.

Acknowledgement: Authors acknowledge the support provided by JNIOSH in conducting the experiments. 